\title{
Imaging the Role of Multinucleate Pancreatic Cancer Cells and Cancer-Associated Fibroblasts in Peritoneal Metastasis in Mouse Models
}

\author{
KOSUKE HASEGAWA ${ }^{1}$, ATSUSHI SUETSUGU ${ }^{1,2,3}$, MIKI NAKAMURA ${ }^{1}$, \\ TAKURO MATSUMOTO ${ }^{1}$, HITOMI AOKI ${ }^{1}$, TAKAHIRO KUNISADA ${ }^{1}$, MASAHITO SHIMIZU ${ }^{1}$, \\ SHIGETOYO SAJI ${ }^{1}$, HISATAKA MORIWAKI ${ }^{1}$ and ROBERT M. HOFFMAN ${ }^{2,3}$ \\ ${ }^{1}$ Gifu University Graduate School of Medicine, Gifu, Japan; \\ ${ }^{2}$ AntiCancer, Inc., San Diego, CA, U.S.A.; \\ ${ }^{3}$ Department of Surgery, University of California San Diego, San Diego, CA, U.S.A.
}

\begin{abstract}
Background/Aim: The interaction between pancreatic-cancer cells and stromal cells in the tumor microenvironment (TME) is of particular importance in cancer progression and metastasis. The present report demonstrates the role of cancer-associated fibroblasts (CAFs) and multinucleate pancreatic-cancer cells in peritoneal metastasis. Materials and Methods: An orthotopic mouse model of pancreatic cancer was established with the human pancreatic cancer cell line BxPC3, which stably expresses green fluorescent protein (GFP). Results: BxPC3-GFP cells formed peritoneal metastases by week 18 after orthotopic implantation. Using an Olympus FV1000 confocal microscope, multinucleated cancer cells were frequently observed in the peritoneal metastases. The primary pancreatic tumor and peritoneal-metastases were harvested, cultured and then transplanted subcutaneously. Subcutaneous tumors established from peritoneal-metastatic cells were larger than subcutaneous tumors established from primary-tumor cells. Subcutaneous tumors of each type were subsequently cultured in vitro. CAFs were observed growing out from the tumors established from peritoneal-metastatic cells, but not the tumors established from
\end{abstract}

This article is freely accessible online.

Correspondence to: Atsushi Suetsugu, MD, Ph.D. Department of Gastroenterology, Gifu University, Graduate School of Medicine, Gifu, Japan, 1-1 Yanagido, Gifu 501-1194, Japan. Tel: +81-58-2306308, Fax: +81 582306310, e-mail: asue@gifu-u.ac.jp or Robert M. Hoffman, Ph.D., AntiCancer, Inc., 7917 Ostrow Street, San Diego, CA 92111, U.S.A. Tel: +1 8586542555, Fax: +1 8582684175, email: all@anticancer.com

Key Words: Pancreatic cancer, nude mice, orthotopic, peritoneal metastasis, cancer-associated fibroblasts, green fluorescent protein, imaging. the primary cancer. Conclusion: The results of the present study suggest that multi-nucleated cancer cells and CAFs were related to peritoneal metastasis of pancreatic cancer.

Pancreatic cancer is characterized by a thick desmoplastic stromal matrix comprising abundant fibroblasts and immune cells that play an important role in cancer progression, metastasis, immunosuppression and resistance to chemotherapy $(1,2)$.

We previously reported color-coded imaging of cell dynamics in the tumor microenvironment (TME) including the interaction between cancer cells and stromal cells (3-5). The tumors acquired brightly-fluorescent stromal cells from transgenic host mice expressing a different color fluorescent protein from the cancer cells in the tumor. The stromal cells were stably associated with the tumors through multiple passages. The colored fluorescent protein-expressing stromal cells included cancer-associated fibroblasts (CAFs) and tumor-associated macrophages (TAMs) (3).

Liver metastases and disseminated peritoneal metastases maintained the fluorescent-protein-expressing stroma from the primary tumor and possibly recruited additional fluorescent protein-expressing stroma, resulting in their very bright fluorescence. The fluorescent protein-expressing stroma included CAFs and TAMs in both the primary and metastatic tumors (6).

With high-resolution intravital imaging afforded by the Olympus FV1000 confocal microscope, the interaction of pancreatic cancer cells expressing green fluorescent protein (GFP) in the nucleus and red fluorescent protein (RFP) in the cytoplasm and RFP-expressing pancreatic stellate cells could be clearly imaged in the liver and other metastases, suggesting that stellate cells participate in metastasis formation (5).

In the present study, we developed a nude-mouse orthotopic model of pancreatic cancer (7-12) resulting in peritoneal metastasis with the human pancreatic cancer cell 
A

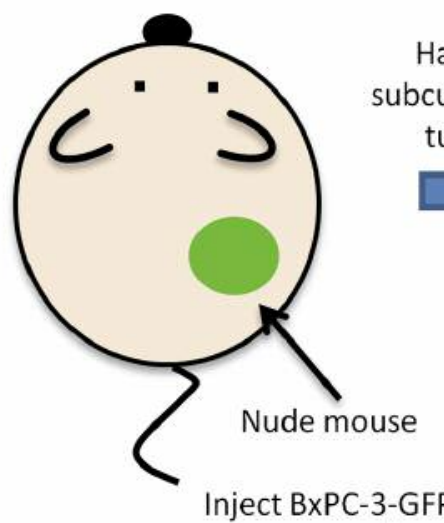
cancer cells
Culture of

peritoneal

metastasis cells

(No 1) and

primary cells

cutaneous

tumor

Peritoneal

metastasis

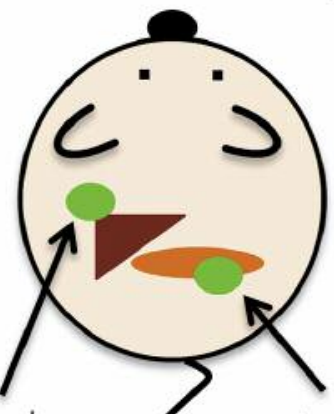

Orthotopic implantation

(No 2)
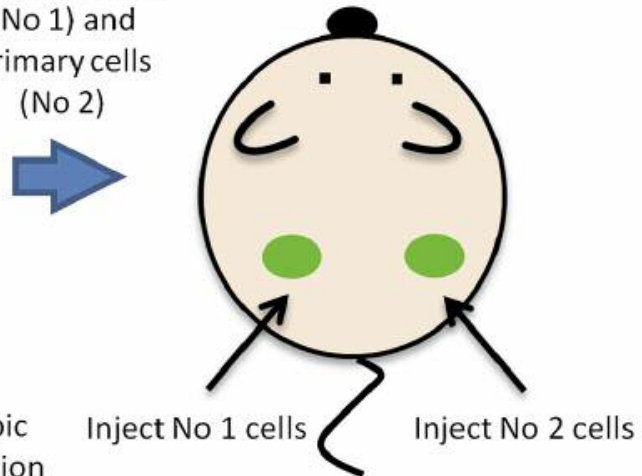

in pancreas

B
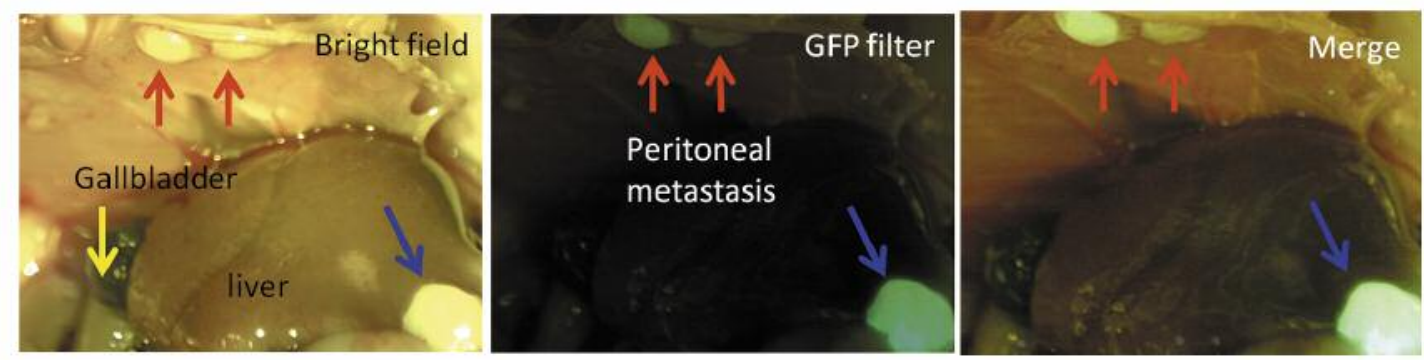

C
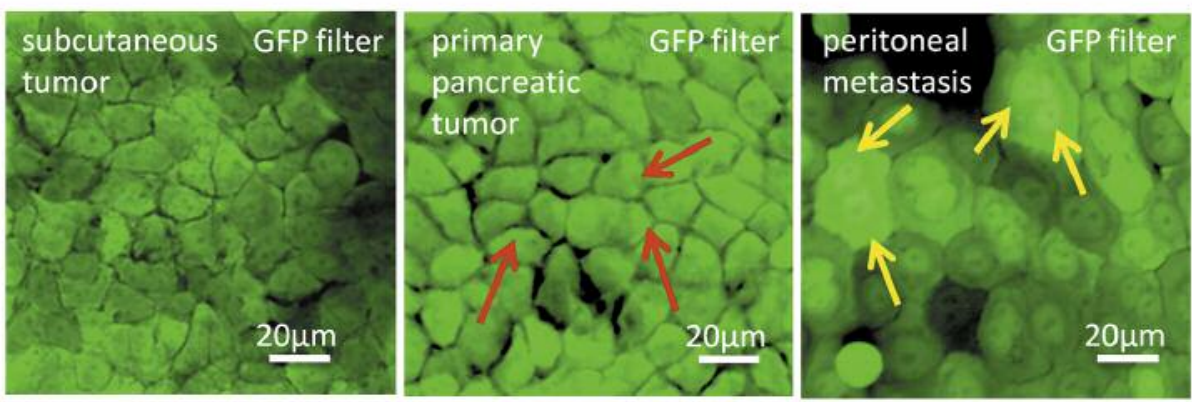

D
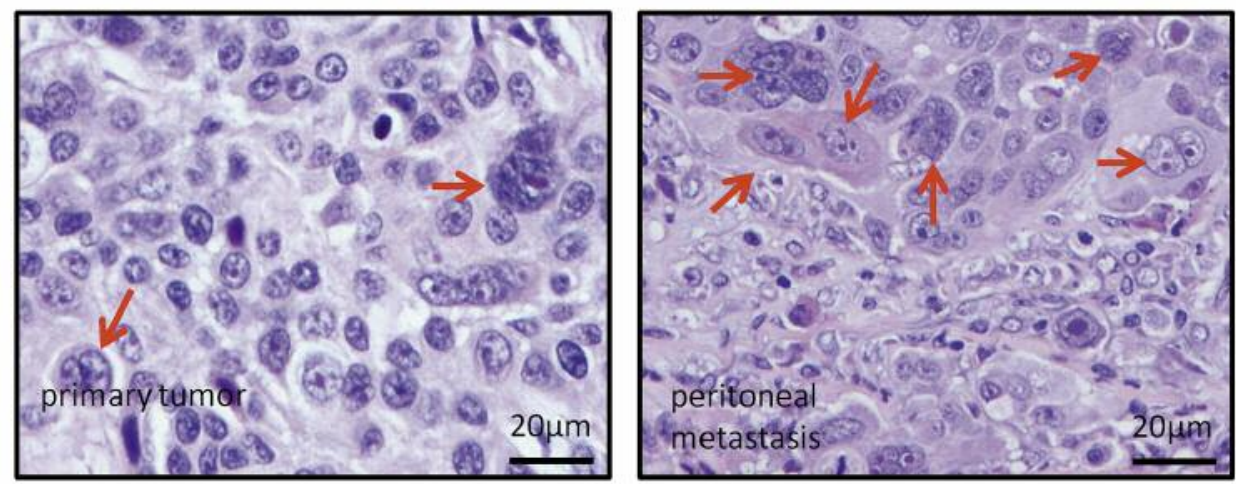

Figure 1. Continued 


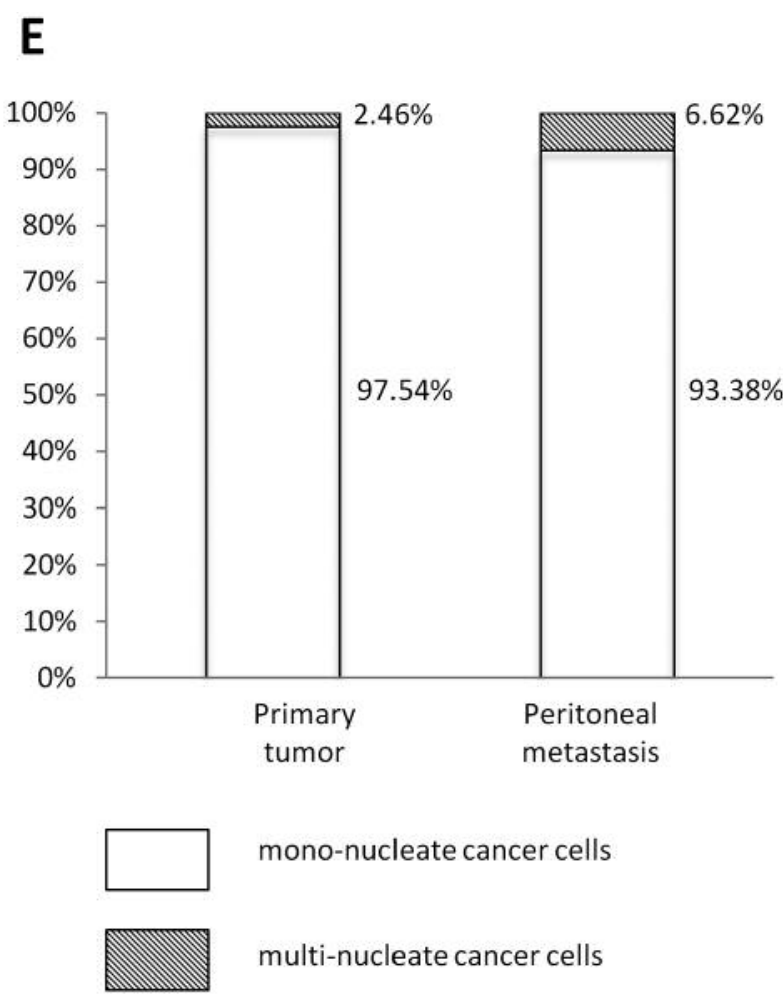

Figure 1. (A) Schematic representation of pancreatic cancer orthotopic xenograft. 35 days after subcutaneous injection of BxPC3-GFP cells, subcutaneous tumors were established. Subcutaneous tumors were harvested, minced into fragments and orthotopically transplanted into the pancreas tail of nude mice. 18 weeks after tumor transplantation, peritoneal metastasis lesions were observed. Black arrows indicate BxPC3-GFP tumors. (B) Bright-field and fluorescence image of the primary tumor and peritoneal metastases. Red arrows indicate peritoneal metastases. Blue arrow indicates the primary pancreatic tumor. Yellow arrow indicates gallbladder. (C) High-magnification fluorescence image of BxPC3-GFP tumors. Left panel shows a subcutaneous tumor. Middle panel shows a primary orthotopic pancreatic tumor. Right panel shows a peritoneal metastasis. Red arrows indicate mono-nuclear cancer cells. Yellow arrows indicate multi-nucleate cancer cells $($ Bar $=20 \mu \mathrm{m})$. (D) Hematoxylin and eosin $(H \& E)$ stained section of BxPC3-GFP tumors. Left panel shows the primary orthotopic pancreatic tumor tissue. Right panel shows the peritoneal metastasis. Red arrows indicate multi-nucleated cells. (E) Five randomly-selected, low-magnification visual fields were quantified for the number of mono-nuclear and multi-nucleate cancer cells. The frequency of each cell type in the primary orthotopic pancreatic tumor and peritoneal metastases are plotted in the bar graphs $(p=0.0003)$.

line (BxPC3), which stably expresses GFP (BxPC3-GFP), in order to further understand the interaction of pancreatic cancer cells and stromal cells in the TME.

\section{Materials and Methods}

Cell line and culture condition. The human pancreatic cancer cell line $\mathrm{BxPC} 3$ was engineered to stably express green fluorescent protein (GFP) (BxPC3-GFP) (13-15). The cells were maintained in RPMI
1640 medium (Gibco-BRL, Grand island, NY, USA). supplemented with $10 \%$ heat-inactivated fetal bovine serum (FBS) (Gibco-BRL) and $1 \%$ penicillin and streptomycin (Gibco-BRL). The cell line was cultured in a humidified atmosphere containing $5 \% \mathrm{CO}_{2}$ at $37^{\circ} \mathrm{C}$.

Pancreatic cancer orthotopic BxPC3-GFP model. Six-week-old nude mice were used as the host. BxPC3-GFP cells were harvested by trypsinization and washed three times with cold serum-free medium, then re-suspended in serum-free RPMI 1640 medium. BxPC3-GFP cells $\left(2.0 \times 10^{6}\right)$ were then injected subcutaneously in 3 nude mice and formed tumors by day 35 after injection. The subcutaneous tumors were harvested and divided into $3 \mathrm{~mm}^{3}$ fragments and orthotopically transplanted to the pancreas tail of BALB/c nude mice, using 5-0 nylon surgical sutures (7-12). Metastases were observed 18 weeks after transplantation (Figure 1A). All surgical procedures were performed with the animals anesthetized by subcutaneous injection of $100 \mathrm{mg} / \mathrm{kg}$ ketamine.

Subcutaneous injection of primary pancreatic tumors and peritoneal metastasis. The primary pancreatic tumor and peritoneal metastases were harvested and the cells were cultured. Pancreatic cancer cells $\left(2.0 \times 10^{6}\right)$ from each source were injected subcutaneously in the right- and left-side back flank, respectively, of BALB/c nude mice. Thirty five days after injection, cells from each source formed subcutaneous tumors.

Tumor imaging. The SZX7 microscope and FV1000 confocal microscope, both from Olympus Corp. (Tokyo, Japan) and Dino-lite digital fluorescence microscope (AM4113T-GFBW Dino-Lite Premier; AnMo Electronics Corp, Hsinchu, Taiwan) were used for intravital and ex vivo imaging. The BX53 microscope (Olympus Corp) was used for histological sections.

Histology. Tumors were prepared for histological analysis using hematoxylin and eosin (H\&E) staining using previously published protocols (16).

Statistical analysis. A two-sided $t$-test was used to determine statistical significance. A $p$-value of $\leq 0.05$ was considered significant.

Study approval. All experiments were conducted in accordance with the Institutional Guidelines of Gifu University and were approved by the Animal Research Committee and the Committee on Living Modified Organisms of Gifu University (approval number 26-37).

\section{Results and Discussion}

Establishment of the pancreatic cancer orthotopic model. Peritoneal metastasis was observed 18 weeks after transplantation (Figure 1B). The primary pancreatic tumors and peritoneal metastases were clearly observed in both bright field and by fluorescence microscopy (Figure 1B, C). Both the primary tumor and peritoneal metastasis brightly expressed GFP. Peritoneal metastasis had multiple foci.

Cell types cultured from the original subcutaneous tumor, primary orthotopic tumor and peritoneal metastasis. 
A
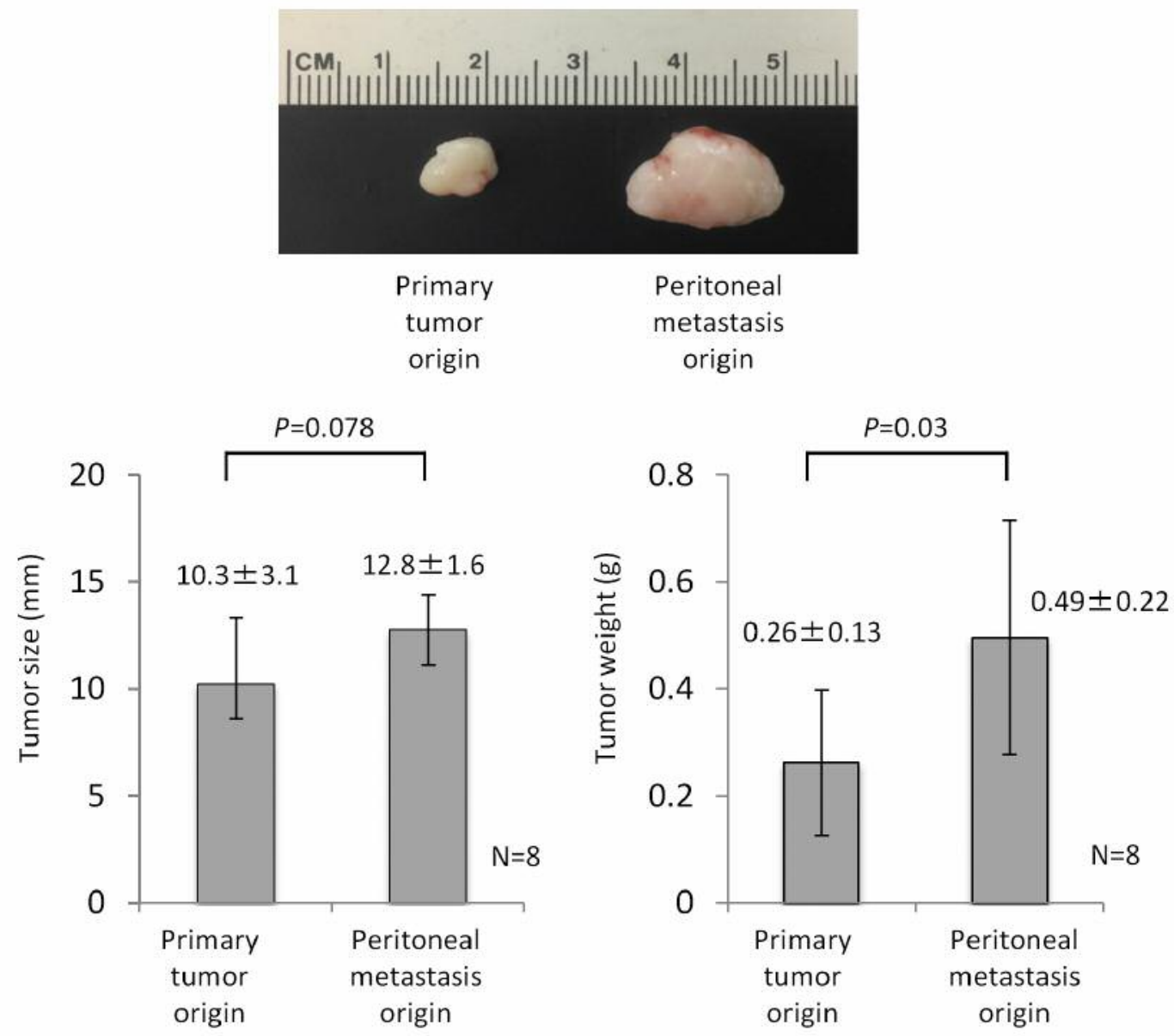

B
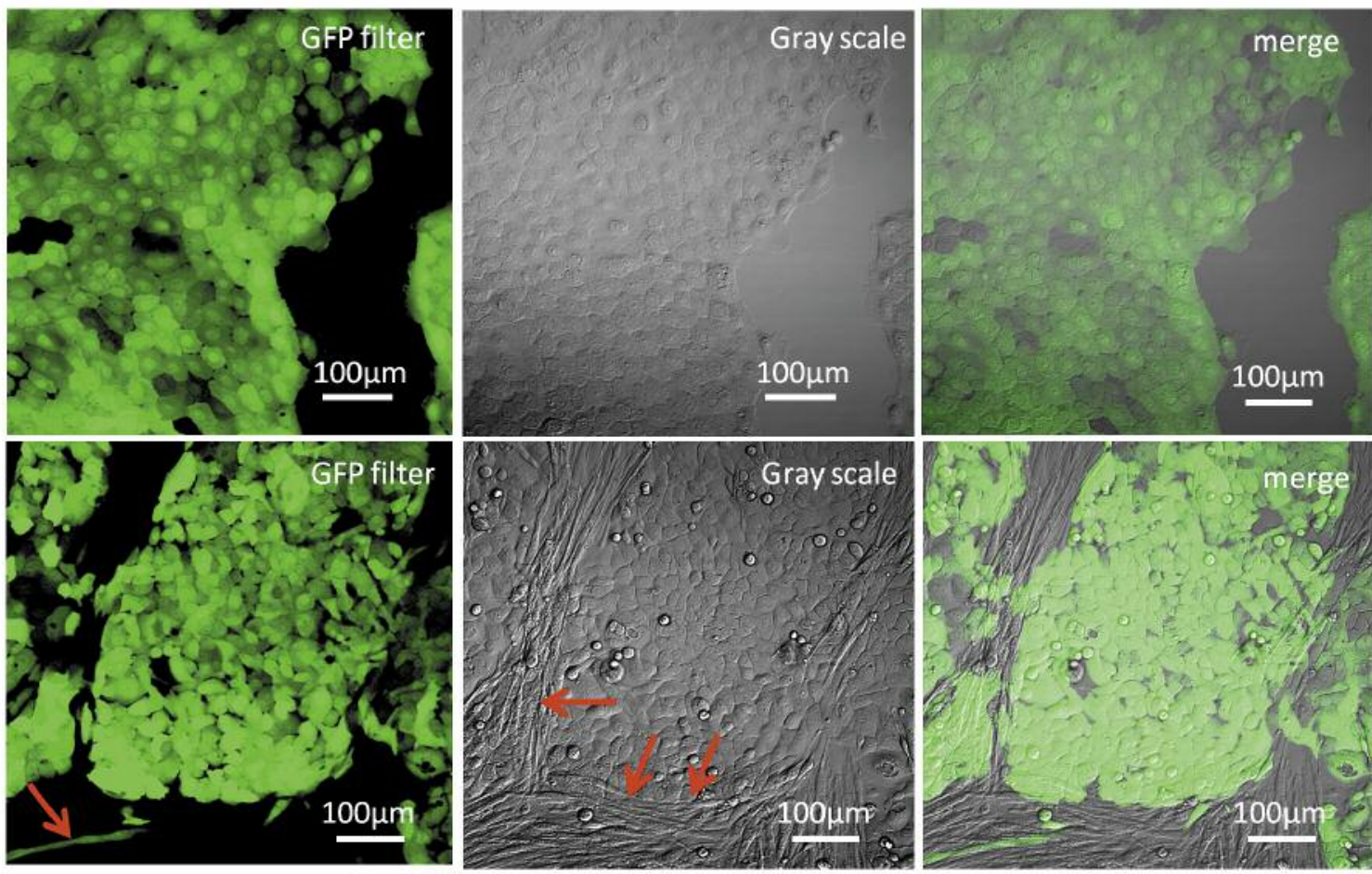
Abnormal multinucleate cancer cells with a large cytoplasm were observed growing from the peritoneal metastases (Figure 1D). These abnormal cancer cells were not observed among the original BxPC3-GFP cells cultured before subcutaneous injection in mice and at a lower amount among cells cultured from the primary orthotopic tumor compared to cells grown from the peritoneal metastases $(p=0.0003)$ (Figure 1E). Multi-nucleate cancer cells were also observed in a metastasis of a colon cancer cell line (17). These multinucleated cells may have resulted from cell fusion or other means by which 2 or more cells can recombine their genetic material and may contribute to greater potential of tumor progression and metastasis (18).

Tumorigenic potential of cells growing out from subcutaneous and the peritoneal metastasis. Cells that grew from subcutaneous tumor and peritoneal metastasis were separately implanted subcutaneously. Tumors that grew from cells derived from the peritoneal metastasis were significantly larger than cells derived from the subcutaneous tumor (Figure 2A).

CAFs found in the peritoneal metastasis. The excised primary pancreatic tumor cells and peritoneal metastasis were cultured for 18 weeks. Large numbers of fibroblasts were observed among the cells growing from the peritoneal metastasis cells but not the primary orthotopic tumor cells (Figure 2B). Therefore, these CAFs appeared to be related to tumor progression and metastasis $(19,20)$.

\section{Conclusion}

BxPC3-GFP cells formed peritoneal metastasis by week 18 after orthotopic implantation. Using an Olympus FV1000 confocal microscope, multi-nucleate cancer cells were observed frequently in the peritoneal metastasis. The primary pancreatic tumor and peritoneal-metastasis were harvested and cultured and each transplanted subcutaneously.

\section{$\leftarrow$}

Figure 2. (A) Cells cultured from an orthotopic tumor (Figure 1A, No 1) and peritoneal metastasis (No 2), were implanted subcutaneously. The comparison of tumor size of each type and weight after 35 days are shown to the bar graph. (B) Cells cultured from the subcutaneous tumor derived from the primary pancreatic tumor or from a subcutaneous tumor derived from a peritoneal metastasis. Upper panels show cells growing from subcutaneous tumors derived from the primary pancreatic tumor. Lower panels show cells cultured from the subcutaneous tumor derived from the peritoneal metastasis. Left panels show GFP fluorescence images. Middle panels show bright field images. Right panels show merged images. Red arrows indicate cancer-associated fibroblasts $(C A F s)$. CAFs were only observed growing from the tumor that originated from cells in the peritoneal metastasis $($ Bar $=100 \mu \mathrm{m})$.
Subcutaneous tumors established from peritoneal metastatic cells were larger than subcutaneous tumors established from the primary tumor cells. Subcutaneous tumors of each type were then cultured in vitro. CAFs and multi-nucleate cancer cells, were observed growing out from the tumors established from peritoneal metastatic cells and to a lesser extent from tumors established from the primary cancer. The results of the present study suggest that multi-nucleated cancer cells and CAFs were related to peritoneal metastasis of pancreatic cancer. Multi-nucleate cancer cells may arise by recombination with other cell types $(18,21)$.

\section{Conflicts of Interest}

None of the Authors have any conflict of interest in regard to this study.

\section{References}

1 Erkan M, Hausmann S, Michalski CW, Fingerle AA, Dobritz M, Kleeff $\mathrm{J}$ and Friess $\mathrm{H}$ : The role of stroma in pancreatic cancer: diagnostic and therapeutic implications. Nat Rev Gastroenterol Hepatol 9: 454-467, 2012.

2 Neesse A, Krug S, Gress TM, Tuveson DA and Michl P: Emerging concepts in pancreatic cancer medicine: targeting the tumor stroma. Onco Targets Ther 7: 33-43, 2013.

3 Hoffman RM and Bouvet M: Imaging the microenvironment of pancreatic cancer patient-derived orthotopic xenografts (PDOX) growing in transgenic nude mice expressing GFP, RFP, or CFP. Cancer Letters 380: 349-355, 2016.

4 Suetsugu A, Katz M, Fleming J, Moriwaki H, Bouvet M, Saji S and Hoffman RM: Multi-color palette of fluorescent proteins for imaging the tumor microenvironment of orthotopic tumorgraft mouse models of clinical pancreatic cancer specimens. J Cell Biochem 113: 2290-2295, 2012.

5 Suetsugu A, Snyder CS, Moriwaki H, Saji S, Bouvet M and Hoffman RM: Imaging the interaction of pancreatic cancer and stellate cells in the tumor microenvironment during metastasis. Anticancer Res 35: 2545-2551, 2015.

6 Suetsugu A, Katz M, Fleming J, Truty M, Thomas R, Saji S, Moriwaki H, Bouvet $M$, and Hoffman RM: Imageable fluorescent metastasis resulting in transgenic GFP mice orthotopically implanted with human-patient primary pancreatic cancer specimens. Anticancer Res 32: 1175-1180, 2012.

7 Fu X, Guadagni F and Hoffman RM: A metastatic nude-mouse model of human pancreatic cancer constructed orthotopically with histologically intact patient specimens. Proc Natl Acad Sci USA 89: 5645-5649, 1992.

$8 \mathrm{Fu} \mathrm{X}$ and Hoffman RM: Human ovarian carcinoma metastatic models constructed in nude mice by orthotopic transplantation of histologically-intact patient specimens. Anticancer Res 13: 283-286, 1993.

9 Fu XY, Besterman JM, Monosov A and Hoffman RM: Models of human metastatic colon cancer in nude mice orthotopically constructed by using histologically intact patient specimens. Proc Natl Acad Sci USA 88: 9345-9349, 1991. 
10 Bouvet M, Wang J, Nardin SR, Nassirpour R, Yang M, Baranov E, Jiang P, Moossa AR and Hoffman RM: Real-time optical imaging of primary tumor growth and multiple metastatic events in a pancreatic cancer orthotopic model. Cancer Res 62: 15341540, 2002.

$11 \mathrm{Fu} \mathrm{X}$, Le P and Hoffman RM: A metastatic-orthotopic transplant nude-mouse model of human patient breast cancer. Anticancer Res 13: 901-904, 1993.

12 Furukawa T, Kubota T, Watanabe M, Kitajima M and Hoffman RM: Orthotopic transplantation of histologically intact clinical specimens of stomach cancer to nude mice: Correlation of metastatic sites in mouse and individual patient donors. Int J Cancer 53: 608-612, 1993.

13 Hoffman RM and Yang M: Subcellular imaging in the live mouse. Nature Protocols 1: 775-782, 2006.

14 Hoffman RM and Yang M: Color-coded fluorescence imaging of tumor-host interactions. Nature Protocols 1: 928-935, 2006.

15 Hoffman RM and Yang M: Whole-body imaging with fluorescent proteins. Nature Protocols 1: 1429-1438, 2006.

16 Igarashi K, Kawaguchi K, Kiyuna T, Murakami T, Miwa S, Nelson SD, Dry SM, Li Y, Singh A, Kimura H, Hayashi K, Yamamoto N, Tsuchiya H, Eilber FC and Hoffman RM: Patientderived orthotopic xenograft (PDOX) mouse model of adult rhabdomyosarcoma invades and recurs after resection in contrast to the subcutaneous ectopic model. Cell Cycle 16: 91-94, 2017.

17 Hasegawa K, Suetsugu A, Nakamura M, Matsumoto T, Aoki H, Kunisada T, Bouvet M, Shimizu M and Hoffman RM: Imaging nuclear-cytoplasmic dynamics in primary and metastatic colon cancer in nude mice. Anticancer Res 36: 2113-2117, 2016.
18 Glinsky GV, Glinskii AB, Berezovskaya O, Smith B,A, Jiang P, Li X-M, Yang M and Hoffman RM: Dual-color-coded imaging of viable circulating prostate carcinoma cells reveals genetic exchange between tumor cells in vivo, contributing to highly metastatic phenotypes. Cell Cycle 5: 191-197, 2006.

19 Vonlaufen A, Joshi S, Qu C, Phillips PA, Xu Z, Parker NR, Toi CS, Pirola RC, Wilson JS, Goldstein D and Apte MV: Pancreatic stellate cells: partners in crime with pancreatic cancer cells. Cancer Res 68: 2085-2093, 2008.

20 Apte MV, Wilson JS, Lugea A and Pandol SJ: A starring role for stellate cells in the pancreatic cancer microenvironment. Gastroenterology 144: 1210-1219, 2013.

21 Tome Y, Tsuchiya H, Hayashi K, Yamauchi K, Sugimoto N, Kanaya F, Tomita $\mathrm{K}$ and Hoffman RM: In vivo gene transfer between interacting human osteosarcoma cell lines is associated with acquisition of enhanced metastatic potential. J Cell Biochem 108: 362-367, 2009. 